\title{
Solar radius determinations obtained with the CCD astrolabe at TUBITAK National Observatory
}

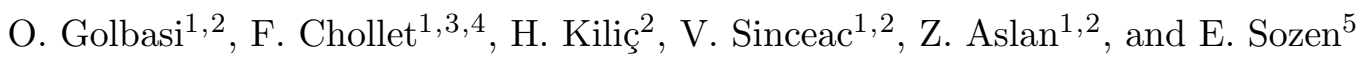 \\ 1 Tubitak National Observatory, Akdeniz University, Antalya, Turkey \\ 2 Akdeniz University, Physics Dept., Antalya, Turkey \\ 3 Paris Observatory, 61 avenue de l'Observatoire, 75014 Paris, France \\ 43 rue de la Source Perdue, 91190 Gif-sur-Yvette, France \\ ${ }^{5}$ Kandilli Observatory, Istanbul, Turkey
}

Received 20 November 2000 / Accepted 20 December 2000

\begin{abstract}
This paper measures the solar radius, using the new Tubitak National Observatory astrolabe as well as data acquisition and reduction procedures. The mean values of the solar radius obtained are slightly lower than the other results to which they are compared. We have compared our results with data obtained since 1981, and show that our results are very homogeneous. We hope to extend these measurements to obtain accurate determinations of solar position.
\end{abstract}

Key words. Sun: general - Sun: oscillations - Sun: photosphere - astrometry - atmospheric effects

\section{Introduction}

Visual observations of the Sun (Laclare et al. 1980; Chollet 1981) show some variations in the solar radius. To improve the quality of this type of measurement in a precise and automated fashion, we have made astrometric observations of the Sun with the new solar astrolabe at Antalya station, National Observatory of Turkey. These measurements aim to estimate the solar radius and, possibly, its variations, using a modern CCD astrolabe with zerodur prisms and a CCD video camera (Sinceac 1998; Sinceac et al. 1998a). Only the automation system, for instrument orientation and horizontality, needs to be installed.

In astrometric observations, the apparent or observed solar radius is always smaller than the true one. In the observed radius, limb darkening is amplified and modified by atmospheric turbulence and transmission. So, the true radius needs to be defined, which we take as (Chollet \& Sinceac 1999) the semi-diameter of a large circular source of light emitting with constant intensity (i.e. without limb darkening). We emphasize here that the observed diameter is always vertical and that each radius determination, obtained by time transit differences, is made at a constant zenith distance.

Send offprint requests to: F. Chollet, e-mail: fernand.chollet@wanadoo.fr

\section{The instrument}

The astrolabe gives two images of the same star which follow two symmetrical trajectories relative to a horizontal line. The principle is to find the instant when the two images of the star are on the same horizontal line. At this instant, the apparent zenith distance of the star is exactly that defined by a prism, equivalent to a double mirror, associated with a mercury surface. Construction of several ceramic glass prisms with different angles allows the possibility to observe at several zenith distances to increase the quantity of daily and annual results. The possible zenith distances attainable in Antalya are $30^{\circ}$ and $60^{\circ}$. A new prism, for the $45^{\circ}$ zenith distance, constructed with the help of the San Fernando Observatory (Sànchez 2000), will be installed next year.

Placed in front of the objective, the optical mount gives two images in the focal plane of the refractor via two separated beams. The separation of the beams introduces a systematic error which is removed by a Wollaston prism placed in the focal plane in order to realign the two beams. The solar observation should be made using a neutral filter (ceramic glass plate covered by a Chrome-Nickel coating with density near 5.5).

The prisms are thermally stable, allowing them to be placed just in front of the telescope objective such that they stay in a fixed position during observations, even when the prism is changed. 
The observer attempts to determine, as well as possible, the true transit time of each solar limb. The observing step acquires a set of about 100 images (with two solar images, due to the two beams) and the exact value of the UTC time of the acquisition for each image. With this set of images the apparent trajectory of each image as a function of time can be reconstructed (Sinceac 1998; Sinceac et al. 1998a; Chollet \& Sinceac 1999). Each CCD frame contains the direct and the reflected images of the Sun.

The video camera has a $576 * 768$ pixel chip with interline transfer. The associated board associated reduces the resolution to $512 * 512$ pixels in two frames ( 256 rows $\times 512$ columns), as in the television standard. On the sky, the resulting pixel covers 0 "'78 (vertical) and 1"10 (horizontal). Taking account of focal length of the astrolabe, the field covered by the camera is approximatly $400^{\prime \prime} \times 280^{\prime \prime}$. The largest visible part of the Sun covers less than $20 \%$ of the total image. The exposure time ranges from $0.1-20 \mathrm{~ms}$.

Due to a defect in the Wollaston prism, these first campaigns (1999 and 2000) should be considered as being carried out by using two different astrolabes. We will see later that, despite this instrumental problem, the results are very similar.

\section{The reductions}

To measure the vertical solar diameter (VSD), we determine, on the two images, the extremities of the VSD along the solar limb. Each image gives the distribution of the apparent solar intensity $I(x, y)$ with respect to the CCD frame defined by the CCD rows and columns.

The subsequent analysis is made so as to obtain successive positions of one of the extremities of the vertical solar diameter in the CCD frame used as a mathematical system of coordinates. As a first approximation to find the reference points on each limb, 3 steps are necessary for each of the two solar images on each of the CCD frames:

- Replace each solar image by a set of points along the limb, by finding the maximum of the derivative of $I\left(x, y_{0}\right)$ along the line $y_{0}$ of the CCD camera;

- Determine the parabolic equation which represents this set of points, and

- Take the position of the extremity of the parabolic curve (the VSD extremity).

The two sets of VSD extremity positions give the trajectories of one of the extremities of the VSD seen directly or reflected by mercury. These coordinates are functions of time: $x_{\mathrm{d}}(t)$ and $y_{\mathrm{d}}(t)$ (direct images), and $x_{\mathrm{r}}(t)$ and $y_{\mathrm{r}}(t)$ (reflected images) in the CCD frame. Knowing these functions, it is possible to determine

- the transit time of the observed extremity of the VSD;

- the correction to the CCD line inclination with respect to the horizon;

- the true extremity of the VSD along the limb, and consequently the corresponding correction to the observed transit time, and
- the pixel sizes on the sky (in arcseconds) along the horizontal and vertical directions.

The transit time of the VSD extremity is obtained when the two coordinates (corrected for the CCD line inclination) $y_{\mathrm{d}}(t)$ of the direct image and $y_{\mathrm{r}}(t)$ of the reflected image are equal. Finally, the comparison of the transit times obtained for each edge of the Sun gives the observed solar diameter. Simple subsequent calculation gives the corrected apparent radius for the unit distance (1 AU).

\subsection{The zenith distance}

The least squares method is used to determine the parabolic curve along the solar edge, as well as the solar trajectories. This method decides during each step whether to reject the lines and/or images via a $2.57 \sigma$ test (Sinceac 1998). This method gives homogeneous results but cannot solve easily the problem of the solar limb definition because of effects of the limb darkening and atmospheric motions. Thus, the apparent solar radius is always smaller than the true one (Rösch et al. 1996; Chollet \& Sinceac 1999). Nevertheless, considering the amount of information obtained during each transit, it may be possible to evaluate these effects and derive the corresponding corrections. We suggest:

- extrapolation of the results of the numerical analysis by a method (to be defined), or

- construction of a model of the solar image, which takes account of all the physical effects.

Using the first method, very interesting correlations have been established between the observed radius and the Fried parameter given by

$r_{0}=8.2510^{5} \cdot D^{-\frac{1}{5}} \cdot \lambda^{\frac{6}{5}} \cdot\left(\sigma^{2}\right)^{-\frac{3}{5}}$

which may be considered as a representation of the atmospheric turbulence (Irbah et al. 1994). Here $D$ is the aperture $^{1}$ of the astrolabe refractor $(m), \lambda$ is the wavelength $(m)$, and $\sigma$ is the standard deviation (") of the linear fit of the observed trajectories ( $r_{0}$ is in meters).

For a given type of instrument, the formula for $r_{0}$ contains only one variable, $\sigma$, which is relatively stable. Consequently, a very large set of measurements is necessary for the extrapolation method.

\section{Results}

A change in the instrumental zenith distance between the observations of the two limbs of the Sun is the only effect able to influence measurement of the diameter. A variable error in the computation of the refraction, a change in the focal length, and/or a change of the prism angle can introduce such an effect.

\footnotetext{
1 The real aperture of the astrolabe is two times $5 \mathrm{~cm} \times 8 \mathrm{~cm}$, due to the two beams created by the entrance prism.
} 


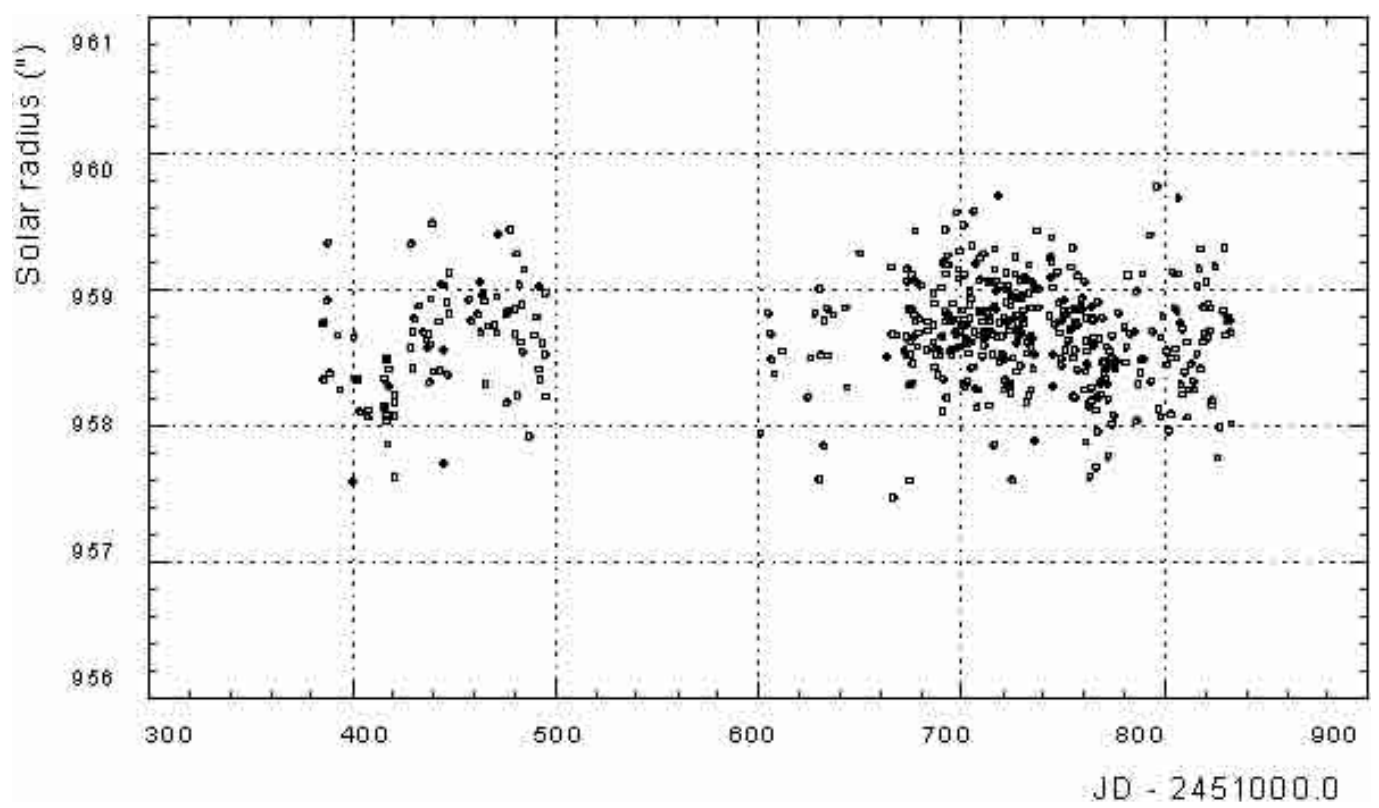

Fig. 1. Antalya Solar radius measurements 1999-2000

As the necessary parameters (pressure and temperature) and the computation of the refraction is performed before and after the solar transit, the error in the refraction evaluation is assumed to be constant during the transit. If the true error is not large, there is no effect on the solar diameter evaluation as long as the error does not change.

The change in focal length is a thermal effect with the same variation for equal changes in temperature. Generally, the effect on the focal length and on the $o b$ served diameter is the same. Consequently, no effect appears between east and west diameter evaluation.

For the same thermal variation, the change in the prism angle is also the same during east and west observations. However, the zenith distance variation of the Sun during the transit is not the same and the east and west results can show systematic differences which allow us to evaluate the instrument quality and stability.

Despite the small number of observations, the mean difference between the east and west radii was computed. We have considered only the data from east and west transits at the same zenith distance on the same day. We obtain:

$\Delta R(E-W)=0$ ". $125 \pm 0$ "' 080 for 1999

and

$\Delta R(E-W)=-0^{\prime \prime} 048 \pm 00^{\prime \prime} 040$ for 2000 .

The total set of data gives:

$\Delta R(E-W)=-0 . .018 \pm 0$ ". 036 for $1999-2000$.
The effect of the change in the prism angle appears noticeable in 1999, but the total set of measures shows that the effect is very small.

\subsection{The observed solar radius}

The observations made in 1999 should be considered as tests of the instrument and the method. However, the results are so similar that using the observations of the two years put together, the correlation between $r_{0}$, (in meter here) and the observed solar radius $R$ was computed to be

$R=959^{\prime \prime} 03-0 !^{\prime \prime m} 016812 \cdot \frac{1}{r_{0}}$.

An ideal atmosphere corresponds to an infinite value for $r_{0}$. When the Fried parameter is extrapolated to $+\infty$, for $\frac{1}{r_{0}}=0$, we obtain the constant term of the preceding relation, so the extrapolated radius $R$ is

$R=9599^{\prime \prime} 03 \pm 0$ 0. $07 \quad(1999-2000)$.

This result may be considered as the value of the solar radius through a perfect atmosphere or above the atmosphere. We see that the precision is not impressive.

The total number of radii obtained, 94 for 1999 and 392 for 2000 are plotted in Fig. 1 and can be found on the TUG server ${ }^{2}$. Table 1 gives the successive means of 20 individual measurements. The mean values for the solar radius obtained for the two years are:

$R=958^{\prime \prime} .60 \pm 0$. 040 for 1999

$R=958^{\prime \prime} 68 \pm 0$ ". 019 for 2000 .

2 http://www.tug.tubitak.gov.tr/ 
Table 1. Solar radius obtained at Antalya: Mean values of 20 individual radius measurements. For clarity, the Fried parameter is given in $\mathrm{cm}$

\begin{tabular}{lccccr}
\hline $\begin{array}{l}\text { Julian day } \\
-2451000.0\end{array}$ & $N$ & $\begin{array}{c}\text { Radius } \\
\left({ }^{\prime \prime}\right)\end{array}$ & $\begin{array}{c}\varepsilon \\
\left({ }^{\prime \prime}\right)\end{array}$ & $\begin{array}{c}r_{\mathrm{o}} \\
(\mathrm{cm})\end{array}$ & Year \\
\hline 399.94 & 20 & 958.39 & 0.08 & 4.3 & 1999 \\
424.73 & 20 & 958.42 & 0.09 & 4.6 & - \\
446.48 & 20 & 958.74 & 0.08 & 4.6 & - \\
473.30 & 20 & 958.82 & 0.08 & 4.6 & - \\
490.41 & 14 & 958.64 & 0.09 & 4.0 & 1999 \\
& & & & & \\
625.49 & 20 & 958.54 & 0.09 & 4.1 & 2000 \\
672.01 & 20 & 958.60 & 0.10 & 4.4 & - \\
682.26 & 20 & 958.76 & 0.06 & 4.5 & - \\
691.86 & 20 & 958.79 & 0.08 & 4.9 & - \\
699.04 & 20 & 958.78 & 0.07 & 5.0 & - \\
& & & & & \\
704.48 & 20 & 958.74 & 0.08 & 4.7 & - \\
711.06 & 20 & 958.76 & 0.06 & 5.0 & - \\
717.60 & 20 & 958.80 & 0.09 & 5.0 & - \\
723.43 & 20 & 958.68 & 0.09 & 4.6 & - \\
728.62 & 20 & 958.82 & 0.05 & 5.1 & - \\
& & & & & \\
734.14 & 20 & 958.70 & 0.09 & 4.8 & - \\
745.64 & 20 & 958.86 & 0.06 & 4.7 & - \\
754.75 & 20 & 958.73 & 0.06 & 4.6 & - \\
762.00 & 20 & 958.49 & 0.08 & 4.9 & - \\
768.40 & 20 & 958.39 & 0.06 & 4.6 & - \\
& & & & & \\
777.56 & 20 & 958.49 & 0.07 & 4.8 & - \\
796.30 & 20 & 958.61 & 0.10 & 4.5 & - \\
810.70 & 20 & 958.61 & 0.09 & 4.7 & - \\
824.25 & 20 & 958.70 & 0.08 & 4.8 & - \\
838.23 & 12 & 958.52 & 0.08 & 4.8 & 2000 \\
\hline & & & & & \\
& & & \\
& 20 & &
\end{tabular}

The dispersion $\sigma$ of the results is practically the same for each year $\left(0^{\prime \prime} 40\right.$ and $\left.0^{\prime \prime} 37\right)$. An extrapolation for $r_{0} \rightarrow \infty$ gives for each year

$R=958^{\prime \prime} .81 \pm 00^{\prime \prime} 08$

and

$R=959^{\prime \prime} 05 \pm 00^{\prime \prime} 07$,

respectively. The entire data set gives, as we see above,

$R=959^{\prime \prime} 03 \pm 0^{\prime \prime} 07$.

A similar subsequent analysis was made using the data of Table 1, giving a different result, $R=959^{\prime \prime} 57$, which, comparing with the results obtained at Cerga (Chollet \& Sinceac 1999) and Rio Observatory (Jilinski et al. 1998; Jilinski et al. 1999; Puliaev 2000) seems to be homogeneous. Taking into account the amount of data in this last analysis, we have to be cautions, and more observations are needed.

\section{Comparison with other results}

Several authors have published mean values for the apparent solar radius, measured with different instruments and methods. These results were compared in a recent paper (Chollet \& Sinceac 1999), (Table 2).

When we investigate the value of the solar radius and its variation, it is necessary to compare not only the results of different instruments at different times but also those obtained during the same period of time. Thus the results must be computed using strictly the same software. The software used was characterized in the Cerga and Paris Observatories (Sinceac 1998; Sinceac et al. 1998a; Chollet 1981; Chollet \& Sinceac 1999) and is used in several stations or has been compared with the other software.

Table 2 shows clearly that different instruments give different results, as judged by their formal errors, even during the same period of time. The observed radii range between 960.53 (Wittmann 1997) to 958."60 (obtained here). Table 2 shows that, despite the variety of instruments, the radius shows a relatively strong decrease (in the order of 0. '04/year).

We can attribute this apparent radius change either to a real variation of the solar radius or to instrumental systematic errors. The possibility of real variation is not excluded.

Our low result may be explained by the fact that we have corrected the instrumental zenith distance for the focal length variations following the results of autocollimation procedures. This parameter plays an important role in the definition (and in the value) of the real zenith distance during observations. Nevertheless, one can see in Table 1 that the mean values, obtained using 20 consecutive individual measurements, show a very good regularity despite the relatively small number of entries.

\section{Conclusion}

Despite probable systematic differences with other results, the solar radius obtained at the Antalya station presents some interesting characteristics. The instrument of Malatya was moved to the Antalya station, so the results obtained at these two stations come, optically speaking, from the same instrument.

One can see that the mean values obtained range from $958^{\prime \prime} .39$ to $9588^{\prime \prime} 86$ (Table 1). A linear regression shows that the results seem to increase very slowly from 1999 to 2000 (About 0!'005/year). Concerning visual observations, we have seen in the past that the first measurements of all the astrolabes resulted in relatively large variations, which disappeared after several months. This was experienced, with large amplitudes, at Cerga, Santiago, and San Fernando stations but not in Antalya. This result alone justifies the use of a CCD camera.

With a new prism which allows us to observe at a $45^{\circ}$ zenith distance, we hope to not only increase the quantity and quality of the results but also to extend the observational program to measurements of positions of the Sun as well as to positions of planets and faint stars. 
Table 2. Some recent results for the apparent solar radius, obtained by different methods and instruments (annual and/or general mean values) in chronological order of observation

\begin{tabular}{|c|c|c|c|c|c|}
\hline Instrument / Method & Remarks & $\mathbf{N}$ & Period & Result & Author / Reference \\
\hline $\begin{array}{l}\text { Solar Diameter Monitor } \\
\text { (photoelectrical) FFTD }\end{array}$ & & & $1981-87$ & $959^{\prime \prime} 68 \pm 0 .{ }^{\prime \prime} 02$ & Brown \& Christensen-Dalsgaard (1998) \\
\hline Limb-Darkening Scans & & 72 & 1981-90 & $959 . .62 \pm 0 . .03$ & Neckel (1995) \\
\hline $\begin{array}{l}\text { Solar Disk Sextant } \\
\text { Fast Fourier Transform } \\
\text { Definition (FFTD) }\end{array}$ & & $\begin{array}{l}1 \text { fly } \\
1 \text { fly }\end{array}$ & $\begin{array}{l}1990 \\
1992\end{array}$ & $\begin{array}{l}959^{\prime \prime} 60 \pm 0^{\prime \prime} 17 \\
959^{\prime \prime} 53 \pm 0 . .09\end{array}$ & $\begin{array}{l}\text { Maier et al. (1992), } \\
\text { Sofia et al. (1994) }\end{array}$ \\
\hline $\begin{array}{l}\text { Solar Visual astrolabe } \\
\text { Malatya Observatory }\end{array}$ & & $\begin{array}{l}34 \\
16\end{array}$ & $\begin{array}{l}1993 \\
1994\end{array}$ & $\begin{array}{l}959^{\prime \prime} .51 \pm 0^{\prime \prime} 09 \\
959^{\prime \prime} 38 \pm 0^{\prime \prime} 15\end{array}$ & $\begin{array}{r}\text { Golbasi et al. (2000) } \\
-\end{array}$ \\
\hline $\begin{array}{l}\text { Solar Visual Astrolabe } \\
\text { Malatya Observatory }\end{array}$ & 2 prisms & 170 & 1993-96 & $959^{\prime \prime} 44 \pm 0.0^{\prime \prime} 05$ & Kiliç (1998) \\
\hline $\begin{array}{l}\text { Drift-Scan CCD } \\
\text { Drift-Scan Visual }\end{array}$ & & $\begin{array}{l}126 \\
427\end{array}$ & $\begin{array}{l}1996 \\
1996\end{array}$ & $\begin{array}{l}959^{\prime \prime} \cdot 73 \pm 0.0^{\prime \prime} 05 \\
960^{\prime \prime} 53 \pm 0 . .02\end{array}$ & Wittmann (1997) \\
\hline $\begin{array}{l}\text { Solar Visual Astrolabe } \\
\text { Santiago Observatory }\end{array}$ & 2 prisms & $\begin{array}{l}123 \\
120\end{array}$ & $\begin{array}{l}1996 \\
1997\end{array}$ & $\begin{array}{l}959^{\prime \prime} .85 \pm 0.0^{\prime \prime} 03 \\
960^{\prime \prime} 00 \pm 0.0^{\prime \prime} 03\end{array}$ & Noël (1998) \\
\hline $\begin{array}{l}\text { Solar CCD Astrolabe } \\
\text { Variable Angle Prism } \\
\text { without rotating shutter } \\
\text { Rio de Janeiro Observatory }\end{array}$ & $\begin{array}{l}\text { East } \\
\text { West }\end{array}$ & $\begin{array}{l}3500 \\
2600\end{array}$ & $\begin{array}{l}1996-97 \\
1996-97\end{array}$ & $\begin{array}{l}959^{\prime \prime} 20 \pm 0^{\prime \prime} 02 \\
959^{\prime \prime} 14 \pm 00^{\prime \prime} 03\end{array}$ & $\begin{array}{l}\text { Jilinski et al. (1998), } \\
\text { Jilinski et al.(1999) }\end{array}$ \\
\hline $\begin{array}{l}\text { Calern Solar Astrolabe } \\
\text { CCD Derivative } \\
\text { CCD Wavelet + Derivative } \\
\text { CCD Model } \\
\text { CCD Derivative } \\
\text { CCD Derivative } \\
\text { Visual }\end{array}$ & $\begin{aligned} \frac{1}{r_{0}} & \rightarrow 0 \\
\sigma_{\mathrm{t}} & \rightarrow 0 \\
z & \rightarrow 0\end{aligned}$ & $\begin{array}{l}348 \\
348 \\
409 \\
409 \\
\\
592 \\
418\end{array}$ & $\begin{array}{l}1996-97 \\
1996-97 \\
1996-97 \\
1996-97 \\
1996-98 \\
1996-98\end{array}$ & $\begin{array}{l}959^{\prime \prime} .45 \pm 0 . .01 \\
959^{\prime \prime} 53 \pm 0 . .01 \\
959 . .64 \pm 0 . .02 \\
959^{\prime \prime} .63 \pm 0 . .08 \\
959 . .59 \pm 0 . .01 \\
959 . .60 \pm 0 . .01\end{array}$ & $\begin{array}{r}\text { Sinceac (1998) } \\
\text { Irbah (1998) } \\
\text { Chollet \& Sinceac (1999) } \\
\text { Sinceac (1998) } \\
\text { Sinceac (1998) } \\
\text { Laclare et al. (1999) }\end{array}$ \\
\hline $\begin{array}{l}\text { Solar CCD Astrolabe } \\
\text { full pupil, without shutter } \\
\text { San Fernando Observatory }\end{array}$ & optical square & 100 & 1998 & $959^{\prime \prime} 33 \pm 0 .{ }^{\prime \prime} 04$ & Sànchez (1999) \\
\hline $\begin{array}{l}\text { Solar CCD astrolabe } \\
\text { Rio de Janeiro Observatory }\end{array}$ & & $\begin{array}{l}1997 \\
2280\end{array}$ & $\begin{array}{l}1998 \\
1999\end{array}$ & $\begin{array}{l}959^{\prime \prime} \cdot 19 \pm 0.0^{\prime \prime} 03 \\
958^{\prime \prime} .94 \pm 0 . .03\end{array}$ & $\begin{array}{l}\text { Puliaev (2000) } \\
\text { Puliaev (2000) }\end{array}$ \\
\hline $\begin{array}{l}\text { Antalya } \\
\text { Solar CCD astrolabe } \\
\text { CCD derivative } \\
\text { CCD derivative }\end{array}$ & $\frac{1}{r_{0}} \rightarrow 0$ & $\begin{array}{c}94 \\
392 \\
486\end{array}$ & $\begin{array}{c}1999 \\
2000 \\
1999-2000\end{array}$ & $\begin{array}{l}958^{\prime \prime} .60 \pm 00^{\prime \prime} 04 \\
958^{\prime \prime} .68 \pm 00^{\prime \prime} 02 \\
959^{\prime \prime} 03 \pm 00^{\prime \prime} 07\end{array}$ & This paper \\
\hline
\end{tabular}

Acknowledgements. This work was supported by the CNRS (France) and TUBITAK (Turkey) through a two year convention of cooperation. We are grateful to the Akdeniz University and Akdeniz University Faculty of Sciences research funds. The cooperation of the Inönü University is gratefully acknowledged. We are also indebted to the Paris and Cerga (France), and Kandilli (Turkey) Observatories as well as San Fernando Observatory which contributed to the construction and installation of the Antalya station. We have also to thank many colleagues from Turkey and France, and also from Brazil and Spain.

\section{References}

Brown, T. M., \& Christensen-Dalsgaard, J. 1998, ApJ, 500, L195

Chollet, F. 1981, Thèse de Doctorat d'État, Université Pierre et Marie Curie, Paris

Chollet, F., \& Sinceac, V. 1999, A\&AS, 139, 219

Golbasi, O., Kiliç, H., \& Chollet, F. 2000, Turkish J. Phys. 24, (2), 77

Irbah, A. 1998, private communication

Irbah, A., Laclare, F., Borgnino, J., \& Merlin, G. 1994, Solar Phys., 149, 213 
Jilinski, E. G., Puliaev, S. P., Penna, J. L., et al. 1998, A\&AS, Puliaev, S., Penna, J. L., Jilinski, E. G., \& Andrei, A. H. 2000, 130,317 A\&AS, 143, 265

Jilinski, E. G., Puliaev, S., Penna, J. L., Andrei, A. H., \& Laclare, F. 1999, A\&AS, 135, 227

Kiliç, H., 1998, Ph.D. Thesis, Akdeniz University, Antalya

Rösch, J., Rozelot, J. P., Deslandes, H., \& Desnoux, V. 1996 Solar Phys., 165, 1

Laclare, F., Demarcq, J., \& Chollet, F. 1980, C. R. Acad. Sci. Paris Série B, 291, 189

Sànchez, M. 1999, private communication

Sànchez, M. 2000, private communication

Sinceac, V. 1998, Thèse de Doctorat, Observatoire de Paris Acad. Sci. Paris Série B, 327, 645

Maier, E., Twigg, L., \& Sofia, S. 1992, ApJ, 389, 447

Neckel, H., 1995, Solar Phys., 156, 7

Noël, F., 1998, A\&AS, 132, 195

Sinceac, V., Chollet, F., Laclare, F., \& Delmas, C. 1998a, A\&AS, 128, 605

Sofia, S., Heaps, W., \& Twigg, L. W. 1994, ApJ, 427, 1048

Wittmann, A. D. 1997, Solar Phys., 171, 231 